\title{
Killer-cell immunoglobulin-like receptor genotype and haplotype combinations in children treated for acute lymphoblastic leukemia
}

\author{
SYLWIA KOETAN ${ }^{1}$, ANDRZEJ KOETAN ${ }^{1}$, KRYSTYNA SOSZYNSSKA ${ }^{2}$, KAROLINA MATIAKOWSKA ${ }^{3}$, \\ MAEGORZATA MORGUT-KLIMKOWSKA ${ }^{3}$, ELŻBIETA GRZEŚK ${ }^{1}$, GRZEGORZ GRZEŚK ${ }^{4}$, \\ ANNA DĄBROWSKA ${ }^{l}$, ANNA URBANCCZYK ${ }^{l}$, JOANNA KONIECZEK ${ }^{l}$, JAN STYCZYŃSKI ${ }^{l}$, \\ OLGA HAUS ${ }^{3}$, MARIUSZ WYSOCKI ${ }^{1}$
}

'Department of Pediatrics, Hematology and Oncology, Collegium Medicum in Bydgoszcz, Nicolaus Copernicus University in Toruń, Poland ${ }^{2}$ Laboratory of Clinical Genetics, $10^{\text {th }}$ Military Research Hospital and Polyclinic Independent Public Healthcare Centre in Bydgoszcz, Poland ${ }^{3}$ Department and Chair of Clinical Genetics, Collegium Medicum in Bydgoszcz, Nicolaus Copernicus University in Toruń, Poland ${ }^{4}$ Department of Pharmacology and Therapy, Collegium Medicum in Bydgoszcz, Nicolaus Copernicus University in Toruń, Poland

\begin{abstract}
Introduction: Acute lymphoblastic leukemia $(A L L)$ is the most common malignancy diagnosed in children. The factors predisposing to ALL remain mostly unknown. Natural killer (NK) cells are a component of innate immunity. Their role is to eliminate cells that were infected with viruses or underwent a neoplastic transformation. The activity of $N K$ cells is regulated by their activating and inhibitory receptors, inter alia killer-cell immunoglobulin-like receptors (KIRs). The available data about a link between the incidence of ALL and KIR genotype are highly inconclusive, and further research is needed to explain whether such a relationship truly exists. The aim of this study was to analyze KIR genotype and haplotype combinations in children treated for ALL.

Material and methods: The study included 49 children diagnosed with ALL at 1.2-19.8 years of age. The control group was composed of 43 healthy subjects aged between 1.2 and 21.9 years. DNA was isolated using QIAamp DNA Mini kits. KIR genotypes were identified by a polymerase chain reaction $(P C R)$ with sequence-specific primers (SSPs). The analysis also included KIR haplotype combinations: $A A, A B$ and $B B$.

Results: Patients with ALL and controls did not differ significantly in the frequencies of individual KIR genes and haplotypes. However, the overall frequency of all 6 activating KIR genes in patients with ALL was significantly higher than in the controls (24.5\% vs. $4.7 \%, p=0.019)$.

Conclusions: The findings presented here imply that individual KIR genes do not play a significant role in the pathogenesis of ALL. Nevertheless, a higher number of activating KIR genes may constitute a risk factor for this malignancy.
\end{abstract}

Key words: NK cells, ALL, killer immunoglobulin-like receptors (KIRs).

(Cent Eur J Immunol 2021; 46 (2): 210-216)

\section{Introduction}

Acute lymphoblastic leukemia (ALL) is the most common childhood malignancy $[1,2]$. However, the determinants of neoplastic transformation and development of ALL have not been unequivocally identified so far. Potential candidates include genetic factors, immune disorders and environmental conditions. Genetic diseases associated with an increased risk of ALL, including DNA repair defects, as well as Down syndrome, may eventually trigger a neoplastic transformation [3-7]. This malignancy is more likely to develop in carriers of some mutated genes [8]; this stimulated ongoing research on still unidentified genetic determinants of ALL.

Natural killer (NK) cells are a pivotal component of innate immunity. Their function is to eliminate cells that underwent a neoplastic transformation or were infected with intracellular pathogens. NK cells need to be distinguished between normal and pathologically altered cells of

Correspondence: Sylwia Kołtan, DSc, Department of Pediatrics, Hematology and Oncology, Collegium Medicum in Bydgoszcz, Nicolaus Copernicus University in Toruń, Poland, e-mail: s.koltan@ cm.umk.pl Submitted: 15.08.2019; Accepted: 28.12.2020 
the host. This is facilitated by the specialized NK receptor system [9-11].

Killer-cell immunoglobulin-like receptors (KIRs) are an important component of the NK receptor system. These molecules are encoded at chromosome 19q13. The KIR family comprises 15 genes and 2 pseudogenes.

KIR genes show a high degree of allelic polymorphism whose results are visible in a substantial individual variability of KIR genotype. Due to this feature, KIR genes are postulated to be the most rapidly changing fragment of the human genome [12-14].

Depending on the composition of KIR genes on chromosome 19, two haplotypes, A and B, can be identified. These haplotypes differ in the number of activating genes (only one activating gene, 2DS4, within haplotype A vs. a higher number of activating genes within haplotype B) [14-20]. Each human genome contains one haplotypic combination variant: $\mathrm{AA}, \mathrm{AB}$ or $\mathrm{BB}$.

A question arises about a link between the incidence of ALL and KIR genotype. The available data on this matter are highly inconclusive, and further research is needed to explain whether such a relationship truly exists and what the reason is for the discrepancies in the results of previous studies.

The aim of the study was to analyze KIR genotype and haplotype combination in children treated for acute lymphoblastic leukemia according to the ALL IC-BFM 2002 protocol at the Department of Pediatrics, Hematology and Oncology in Bydgoszcz.

\section{Material and methods}

The study included 49 Caucasians, Polish by nationality, who had been diagnosed with ALL at 1.2-19.2 years of age (median age at diagnosis: 5.3 years). The proportion of girls $(53.1 \%)$ in the study group was higher than the percent-

Table 1. Characteristics of patients and the controls

\begin{tabular}{lcc}
\hline Variable & $\begin{array}{c}\text { Patients } \\
\boldsymbol{n}=\mathbf{4 9}\end{array}$ & $\begin{array}{c}\text { Controls } \\
\boldsymbol{n}=\mathbf{4 3}\end{array}$ \\
\hline $\begin{array}{l}\text { Age (diagnosis) } \\
\text { Range (years) }\end{array}$ & $1.1-19.2$ & - \\
\hline Me & 5.3 & - \\
\hline Age (examination) & - & 11.5 \\
\hline Range (years) & -21.9 \\
\hline Me & & \\
\hline Gender & $26(53.1 \%)$ & $23(53.5 \%)$ \\
\hline F & $23(46.9 \%)$ & $20(46.5 \%)$ \\
\hline M & $45(91.8 \%)$ & - \\
\hline Phenotype of ALL & $4(8.2 \%)$ & - \\
\hline Line B & & \\
\hline Line T & & \\
\hline
\end{tabular}

age of boys (Table 1$)$. The vast majority of them $(n=45$, $91.8 \%)$ were diagnosed with B-cell ALL, and $4(8.2 \%)$ with T-cell ALL. All patients were treated according to the ALL IC-BFM 2002 protocol.

The control group was composed of 43 Caucasians, Polish by nationality, aged between 1.2 and 21.9 years (median age: 11.5 years), among them $53.5 \%$ girls and $46.5 \%$ boys (Table 1 ).

A single sample of whole blood (2-3 ml) from each patient and control was collected in an EDTA-coated tube. DNA was isolated using QIAamp DNA Mini kits from QIAGEN (Westburg, Leusden, The Netherlands), according to the manufacturer's instructions published in the $2^{\text {nd }}$ edition of QIAamp DNA Mini And Blood Mini Handbook [21].

KIR genotypes were identified by a polymerase chain reaction (PCR) with sequence-specific primers (SSPs). The complete list of genes identified within the framework of the study is presented in Table 2. All procedures were carried out using KIR Typing Kits from Miltenyi Biotec (Bergisch Gladbach, Germany), in line with the manufacturer's instructions [22].

Table 2. Frequencies of genes encoding individual KIRs

\begin{tabular}{|c|c|c|c|}
\hline Gene & Patients & Controls & $p$ \\
\hline $2 D L 1$ & $48(98.0 \%)$ & $43(100.0 \%)$ & 0.260 \\
\hline $2 D L 2$ & $32(65.3 \%)$ & $24(55.8 \%)$ & 0.352 \\
\hline $2 D L 3$ & $42(85.7 \%)$ & $38(88.4 \%)$ & 0.705 \\
\hline $2 D L 4$ & $49(100.00 \%)$ & $43(100.0 \%)$ & - \\
\hline $2 D L 5$ all & $30(61.2 \%)$ & $24(55.8 \%)$ & 0.599 \\
\hline $2 D L 5 A$ & $25(51.0 \%)$ & $20(46.5 \%)$ & 0.666 \\
\hline $2 D L 5 B$ & $30(61.2 \%)$ & $24(55.8 \%)$ & 0.599 \\
\hline $2 D S 1$ & $28(57.1 \%)$ & $17(39.5 \%)$ & 0.091 \\
\hline $2 D S 2$ & $30(61.2 \%)$ & $25(58.1 \%)$ & 0.763 \\
\hline $2 D S 3$ & $20(48.8 \%)$ & $14(32.6 \%)$ & 0.412 \\
\hline $2 D S 4$ & $46(93.9 \%)$ & $40(93.0 \%)$ & 0.797 \\
\hline $2 \mathrm{DS} 4 \mathrm{del}$ & $40(81.6 \%)$ & $35(81.4 \%)$ & 0.977 \\
\hline 2DS4 ins & $25(51.0 \%)$ & $21(48.8 \%)$ & 0.835 \\
\hline $2 D S 5$ & $23(46.9 \%)$ & $19(44.2 \%)$ & 0.791 \\
\hline $3 D L 1$ & $47(95.9 \%)$ & $40(93.0 \%)$ & 0.541 \\
\hline $3 D L 2$ & $49(100.00 \%)$ & $43(100.0 \%)$ & - \\
\hline $3 D L 3$ & $49(100.00 \%)$ & $43(100.0 \%)$ & - \\
\hline $3 D S 1$ & $28(57.1 \%)$ & $22(51.2 \%)$ & 0.566 \\
\hline $2 D P 1$ & $49(100.00 \%)$ & $43(100.0 \%)$ & - \\
\hline $3 D P 1$ & $49(100.00 \%)$ & $43(100.0 \%)$ & - \\
\hline
\end{tabular}

Frequencies of individual KIR genes were compared with $\chi^{2}$ tests. No statistically significant differences were found in the frequencies of these genes in patients and controls. The most evident, albeit still insignificant, difference was found for the 2DS1 gene $(p=0.091)$. $p$-significance level 


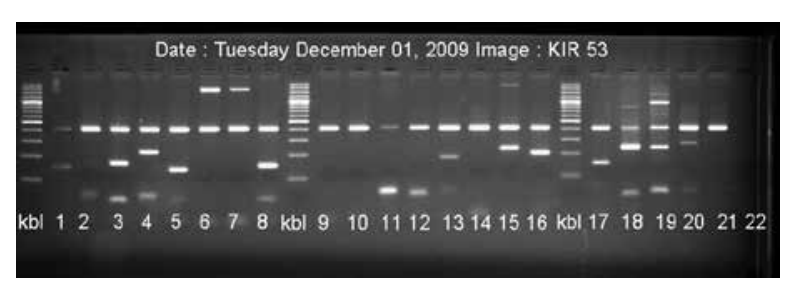

Fig. 1. Representative electrophoretogram of KIR genes on an agarose-coated plate. Numbers of stripes correspond to the following genes: $1-2 D L 1,2-2 D L 2,3-2 D L 3$, $4-2 D L 4,5-2 D L 5$ all, $6-2 D L 5 A, 7-2 D L 5 B, 8-$ 2DS1, 9-2DS2, 10 - 2DS3, 11 - 2DS4del, 12 - 2DS4ins, 13 - 2DS5, 14 - 3DL1, 15 - 3DL2, 16 - 3DL3, 17 - 3DS1, 18 - 2DP1, 19 - 3DP1, 20 - DNA contamination control, 21 - positive control ( $\beta$-actin), 22 - negative control. Interpretation of the result: positive results (number of stripe in parenthesis) - 2DL1 (1), 2DL3 (3), 2DL4 (4), 2DL5all (5), 2DL5A (6), 2DL5B (7), 2DS1 (8), 2DS5 (13), 3DL2 (15), 3DL3 (16), 3DS1 (17), 2DP1 (18), 3DP1 (19). 2D - receptor-encoding genes with two extracellular immunoglobulin-like domains, $3 D$ - receptor-encoding genes with three extracellular immunoglobulin-like domains, $L$ - long transmembrane sequence - inhibitory genes, $S$ - short transmembrane sequence - activating genes, $P$ - pseudogene

Genes for inhibitory receptors, with long intracellular sequences, are designated with the letter $\mathrm{L}$, and genes for activating receptors, with short intracellular sequences, with the letter S. Each gene has two alleles. The only exceptions are $3 D L 1$ and $3 D S 1$, and $2 D L 2$ and $2 D L 3$, considered to be alleles of the same genes [23]. Noticeably, one gene (2DL4) encodes KIR with both an inhibitory and activating function [24, 25].

Table 3. Odds ratios (ORs) with their $95 \%$ confidence intervals and $p$-values

\begin{tabular}{|c|c|c|c|}
\hline Gene & Odd ratio (OR) & $\begin{array}{c}\text { Confidence interval } \\
95 \% \mathrm{CI}\end{array}$ & $p$ \\
\hline $3 D L 1$ & 1.763 & $0.273-11.362$ & 0.548 \\
\hline $2 D L 3$ & 0.789 & $0.227-2.743$ & 0.706 \\
\hline $2 D S 4$ & 1.150 & $0.214-6.170$ & 0.869 \\
\hline $2 D L 2$ & 1.490 & $0.635-3.497$ & 0.353 \\
\hline $2 D L 5$ & 1.250 & $0.537-2.907$ & 0.600 \\
\hline $3 D S 1$ & 1.273 & $0.552-2.934$ & 0.566 \\
\hline $2 D S 2$ & 1.137 & $0.488-2.650$ & 0.763 \\
\hline $2 D S 3$ & 1.429 & $0.600-3.402$ & 0.414 \\
\hline $2 D S 5$ & 1.117 & $0.485-2.573$ & 0.791 \\
\hline $2 D S 1$ & 2.039 & $0.876-4.745$ & 0.094 \\
\hline
\end{tabular}

$\overline{O d d s}$ ratios (ORs) for the co-existence of ALL with individual KIR genes, detected with various frequencies in patients and controls, were determined by means of logistic regression analysis, along with their $95 \%$ confidence intervals. Although no genes with statistically significant ORs were identified, the OR value for $2 D S 1$ was relatively high $(O R=2.039)$.
Some genes are considered to be variants of the same gene. This refers to $2 D L 5$, which may exist in two variants, $2 D L 5 A$ and $2 D L 5 B$, which are separate genes encoded in different loci. The genotyping method used within the framework of this study might detect both the $2 D L 5$ gene (2DL5all) and each of its two variants $[22,26]$. Furthermore, it is suitable for distinguishing between 2DS4del and $2 D S 4 i n s$ [22]. Since the former allele, KIR2DS4del, has no biological function [27], it was not considered as a gene for the functional activating receptor. A representative result of genotyping is presented below (Fig. 1).

Aside from the determination of KIR genotypes, AA, $\mathrm{AB}$ and $\mathrm{BB}$ were identified in line with the following rules: 1. AA haplotype combination - a lack of the following KIR genes: 2DL2, 2DL5A, 2DL5B, 2DS1, 2DS2, 2DS3, $2 D S 5$ and $3 D S 1$.

2. AB haplotype combination - the presence of at least one of the genes mentioned above, along with all the following KIR genes: KIR 2DL1, 2DL3, 2DS4 and $3 D L 1$.

3 . BB haplotype combination - the presence of at least one of the following genes: $2 D L 2,2 D L 5 A, 2 D L 5 B, 2 D S 1$, $2 D S 2,2 D S 3,2 D S 5$ and $3 D S 1$, and a lack of at least one of the following genes: $2 D L 1,2 D L 3,2 D S 4$ and $3 D L 1$ [16].

The statistical analysis of qualitative variables was carried out with 2 tests. Furthermore, odds ratios (ORs) were estimated for some of the study variables. The results of statistical tests were considered significant whenever their $p$-values were below one of the commonly accepted thresholds: $p<0.05, p<0.01$ or $p<0.001$. All calculations were carried out with the Statistica 9.0 package (StatSoft, Poland).

The protocol of the study was approved by the Local Bioethics Committee at the Collegium Medicum in Bydgoszcz.

\section{Results}

KIR genotype was determined in all patients and controls. All subjects carried inhibitory genes, 2DL4, 3DL2 and $3 D L 3$, as well as pseudogenes, $2 D P 1$ and $3 D P 1$. Also the frequencies of other inhibitory genes did not differ significantly between the study groups. It was documented that each of the activating genes in patients with ALL was slightly more frequent compared to the control group: $2 D S 1-57.1 \%$ vs. $39.5 \%, 2 D S 2-61.2 \%$ vs. $58.1 \%, 2 D S 3$ $-40.8 \%$ vs. $32.6 \%, 2 D S 4-93.9 \%$ vs. $93 \%, 2 D S 5-46.9 \%$ vs. $44.2 \%$ (Table 2 ).

We used a logistic regression analysis to calculate odd ratios (ORs) for the association of ALL with the expression of individual KIR genes. Although the OR value for the 2DS1 gene was relatively high, it was of borderline significance $(\mathrm{OR}=2.039, p=0.094)$ (Table 3$)$.

As mentioned above, $2 D S 4$ can occur in two variants: $2 D S 4 d e l$, which does not code a functional receptor, and 2DS4ins, constituting a genetic matrix for the activating re- 
ceptor. Most patients and controls $(93.9 \%$ and $93 \%$, respectively) turned out to be carriers of the $2 D S 4$ gene. However, its inactive form, $2 D S 4 \mathrm{del}$, was the only variant of this gene found in $42.9 \%$ and $48.8 \%$ of patients and controls, respectively. No statistically significant intergroup differences were found in the percentages of 2DS4ins carriers (Table 4). The subgroup of AA haplotype combination carriers included 4/10 patients and 3/9 controls in whom 2DS4del was the only variant of the $2 D S 4$ gene (Table 4); NK cells of such subjects do not synthesize any activating KIRs. The subgroup of participants with a KIR haplotype combination other than AA included only one subject with a single activating gene: a child with $\mathrm{BB}$ haplotype combination who tested negatively for $2 D S 4$ and positively for $2 D S 2$.

Patients with ALL and controls did not differ significantly in terms of $\mathrm{AA}, \mathrm{AB}$ and $\mathrm{BB}$ haplotype combination frequencies. Nevertheless, the frequency of the $A B$ haplotype combination in patients with ALL was slightly higher (61.2\%) and the frequency of the BB haplotype combination slightly lower $(18.4 \%)$ than in the controls $(58.0 \%$ and $21.0 \%$, respectively). The frequencies of the AA haplotype combination in patients and controls were essentially the same $(20.4 \%$ and $21.0 \%$, respectively) (Table 5).

The overall frequency of 6 activating genes in patients with ALL turned out to be significantly higher than in the controls $(24.5 \%$ vs. $4.7 \%, p=0.05)$ (Table 5$) .0$ to 3 activating KIR genes were detected in $23(46.9 \%)$ and $25(58.1 \%)$ patients and controls, respectively, and at least 4 activating genes in $26(53.1 \%)$ and $18(41.9 \%)$, respectively.

\section{Discussion}

A number of population-based studies have been conducted since the implementation of KIR genotyping methodology. The results of these studies vary considerably, especially in terms of individual gene and haplotype combination frequencies.

The most comprehensive source of information about KIR genotypes and haplotypes is presented in the online database available at www.allelefrequencies.net [28]. It also includes a dataset for 690 individuals from Poland [28]. Another source of information is the results of a study published in 2006 by Łuszczek et al. [29]. The frequencies of individual KIR genes in both datasets are essentially similar and generally consistent with our findings.

All the study subjects carried inhibitory genes, $2 D L 4$, $3 D L 2$ and $3 D L 3$, and more than $95 \%$ of them were also carriers of the $2 D L 1$ gene (the frequency of this gene determined by Łuszczek et al. was slightly lower, 91.4\%) [28, 29].

The frequencies of the $2 D L 3$ gene in our patients $(85.7 \%)$ and controls $(88.4 \%)$ did not differ significantly. While slightly lower than in both previously examined Polish populations ( $91.6 \%$ and $91.4 \%$ ), the frequencies of the $2 D L 3$ gene in our series were similar to those reported for other Caucasian populations (86.8-90.9\%) [28, 29].

2DL2 is a less frequent KIR gene. While its frequency in the controls was similar as in both previously examined Polish populations (ca. 55\%), the percentage of $2 D L 2$ carriers among children with ALL was slightly higher $(65 \%)$.

The frequency of the $2 D L 5$ gene in two previously examined Polish populations was estimated at 50\% and 53\%, respectively [28]. In our study, this gene was detected in $55.8 \%$ and $61.2 \%$ of controls and patients, respectively.

Markedly more evident discrepancies pertain to the frequencies of individual activating genes. The most frequent gene from this group is $2 D S 4$, found in all previously examined Caucasians [28, 29], as well as in more than $90 \%$ of patients and controls participating in our study. The authors of previous studies did not specify which variant of this gene was detected, 2DS4ins or rather 2DS4del.

Table 4. Variants of 2DS4 gene detected in patients and controls overall and in carriers of AA haplotype combination

\begin{tabular}{|c|c|c|c|}
\hline \multirow[t]{2}{*}{ Gene } & All patients & All controls & \multirow[t]{2}{*}{$p$} \\
\hline & $n=49(100.0 \%)$ & $n=43(100.0 \%)$ & \\
\hline $2 D S 4$ & $46(93.9 \%)$ & $40(93.0 \%)$ & 0.797 \\
\hline 2DS4 del (exclusively) & $21(42.9 \%)$ & $19(48.8 \%)$ & 0.898 \\
\hline 2DS4 ins (exclusively) & $6(12.2 \%)$ & $5(11.6 \%)$ & 0.928 \\
\hline $2 D S 4$ del and ins & $19(38.8 \%)$ & $16(37.2 \%)$ & 0.877 \\
\hline \multirow[t]{2}{*}{ Gene } & Patients with AA haplotype combination & Controls with AA haplotype combination & \multirow[t]{2}{*}{$p$} \\
\hline & $n=10(100.0 \%)$ & $n=9(100.0 \%)$ & \\
\hline 2DS4 del (exclusively) & $4(40.0 \%)$ & $3(33.3 \%)$ & 0.770 \\
\hline 2DS4 ins (exclusively) & $0(0.0 \%)$ & $1(11.1 \%)$ & 0.957 \\
\hline 2DS4 del and ins & $6(60.00 \%)$ & $5(55.6 \%)$ & 0.788 \\
\hline
\end{tabular}


Table 5. Distribution of KIR haplotype combination and numbers of activating and inhibitory genes

\begin{tabular}{|c|c|c|c|}
\hline \multirow[t]{2}{*}{ Haplotype/No. of genes } & Patients & Controls & \multirow[t]{2}{*}{$p$} \\
\hline & $n=49(\%)$ & $n=43(\%)$ & \\
\hline \multicolumn{4}{|l|}{ Haplotype combination } \\
\hline AA & $10(20.4 \%)$ & $9(21.0 \%)$ & 0.950 \\
\hline $\mathrm{AB}$ & $30(61.2 \%)$ & $25(58.0 \%)$ & 0.763 \\
\hline BB & $9(18.4 \%)$ & $9(21.0 \%)$ & 0.757 \\
\hline \multicolumn{4}{|l|}{ Number of activating genes } \\
\hline $0^{*}$ & $4(8.2 \%)$ & $3(7.0 \%)$ & 0.857 \\
\hline $1 * *$ & $7(14.3 \%)$ & $6(13.9 \%)$ & 0.940 \\
\hline 2 & $6(12.2 \%)$ & $6(13.9 \%)$ & 0.808 \\
\hline 3 & $6(12.2 \%)$ & $10(23.3 \%)$ & 0.164 \\
\hline 4 & $8(16.3 \%)$ & $6(13.9 \%)$ & 0.751 \\
\hline 5 & $6(12.2 \%)$ & $10(23.3 \%)$ & 0.164 \\
\hline 6 & $12(24.5 \%)$ & $2(4.7 \%)$ & 0.019 \\
\hline \multicolumn{4}{|l|}{ Number of inhibitory genes } \\
\hline 8 & $18(36.7 \%)$ & $11(25.6 \%)$ & 0.249 \\
\hline 7 & $16(32.7 \%)$ & $18(41.8 \%)$ & 0.361 \\
\hline 6 & $15(30.6 \%)$ & $14(32.6 \%)$ & 0.841 \\
\hline
\end{tabular}

$0^{*}-2 D S 4$ gene in del variant which does not encode any receptor, $1 * *-2 D S 4$ gene in ins variant, encoding activating receptor $2 D S 4$

Frequencies of $A A, A B$ and $B B$ haplotypes combination and numbers of activating and inhibitory genes detected in patients and controls were compared with $\chi^{2}$ tests. The overall frequency of 6 activating genes in children with acute lymphoblastic leukemia (ALL) turned out to be significantly higher than in the controls. p-significance level

This information is important since only 2DS4ins encodes a functional KIR. In our present study, the frequency of the non-functional variant, $2 \mathrm{DS} 4 \mathrm{del}$, in both patients and controls was higher $(81.6 \%$ and $81.4 \%$, respectively) than the frequency of the functional gene, 2DS4ins ( $51 \%$ and $48.8 \%$, respectively).

The most striking differences refer to the frequency of the $2 D S 1$ gene. In our study, this gene was markedly more often detected in patients with ALL than in the controls (57.1\% and $39.5 \%$, respectively); the difference was not statistically significant. Łuszczek et al. [29] found 2DS1 in $48.6 \%$ of subjects from the Polish population, and the frequency of this gene in Poles whose data are included in the online database was $40.9 \%$ [28]. The frequency of this gene in our control group was essentially similar as in the latter larger population $(n=690)$ [28].

Also the frequency of another activating gene, 2DS2, in our patients with ALL (61.2\%) was higher, compared both to the controls $(58.1 \%)$ and to previously examined Polish populations, either that included in the online database (56.2\%) [28] or the one examined by Euszczek et al. $(50.3 \%)$ [29]. However, the analysis of other records demonstrated that the frequency of this gene varies across populations, from more than $80 \%$ to less than $10 \%$ [28].

The frequency of another gene, $2 D S 3$, in patients with ALL (40.8\%) was higher than in the controls (32.6\%). Even lower frequencies of this gene (24.8\% and $29.1 \%)$ were documented in both previously examined populations $[28,29]$. Also the frequencies of this gene vary from population to population, from more than $70 \%$ to no more than $20 \%$ [28].

The frequencies of the $2 D S 5$ gene in our patients $(46.9 \%)$ and controls $(44.2 \%)$ were higher than in previously examined Polish populations (24.1\% and 32.6\%) $[28,29]$. While this gene is usually detected in $20-79 \%$ of people, its frequency in a Brazilian Amazon population approximated $90 \%$, and it was not detected in any Bulgarian [28].

All participants of our study carried two pseudogenes, $2 D P 1$ and $3 D P 1$. However, both $2 D P 1$ and $3 D P 1$ were previously found in $100 \%$ of examined individuals from other Caucasian populations, e.g. in Germans [28, 29].

The frequencies of some KIR genes, especially activating ones, may vary considerably across populations. Furthermore, this heterogeneity refers not only to geographically "distant" populations but also to those living in close vicinity.

The determination of $\mathrm{AA}, \mathrm{AB}$ and $\mathrm{BB}$ haplotype combination frequencies faces a number of challenges. We used the method proposed by Middleton et al., which is suitable to distinguish between AA, AB and BB haplotype combinations on the basis of genotyping performed solely in a study group [15]. After verification against true haplotype combination frequencies determined in family 
studies, the error rate of this method turned out to be very low [15]. However, the only haplotype combination that can be identified unequivocally using this approach is AA. All other variants contain at least one B haplotype, and therefore they are currently often referred to as Bx [28].

In our study, the frequencies of AA haplotype combination in patients and controls were $20.4 \%$ and $20.95 \%$, respectively. These proportions are slightly lower than the frequency of this haplotype combination in the population of less than 700 Polish people whose data are included in the online database (25.5\%) [28]. Our patients and controls did not differ significantly in terms of $\mathrm{AB}$ and $\mathrm{BB}$ haplotype combination frequencies.

Our series of patients with ALL was highly homogeneous: it included only Caucasians, Polish by nationality, diagnosed with acute lymphoblastic leukemia in childhood (except for one patient diagnosed at 20 years of age). Also the control group was composed solely of Caucasians with Polish nationality. The hereby documented frequencies of individual KIR genes closely resemble those determined in two previous large analyses of Polish people; the only differences worth mentioning pertain to slightly higher frequencies of individual activating genes in patients with ALL.

KIR genotypes in children with ALL have been a subject of a few published studies. The results of these studies are highly inconclusive [30-34]. The differences in the frequencies of activating genes in our patients and controls were markedly less evident than in a similar Canadian population. While in our study, virtually all activating genes were detected slightly more often in subjects with ALL than in the controls, their frequencies in Canadian patients were significantly lower than in healthy children [30]. Moreover, we found that the overall frequency of all 6 activating genes was higher in children with ALL than in healthy controls. This observation is in line with a clinical confirmation of the role of activating KIRs when paired with putative HLA ligands in hematopoietic stem cell transplantation in malignant patients [35].

In 2012, Babor et al. [36] published the results of a German study of children with ALL; the authors unequivocally excluded a relationship between the presence of KIRs and increased risk of this malignancy. In 2015, Oevermann et al. [33] confirmed these findings in another group of 328 children with ALL.

Our findings are consistent with the abovementioned results since we also did not find an association between the risk of ALL and the frequencies of individual activating and inhibitory genes and their haplotype combinations $[33,36]$. Most activating genes were detected slightly more often in patients with ALL than in the controls, and the overall frequency of all 6 genes from this group turned out to be significantly higher in the former group.

Our findings are in partial agreement with the results published by Indian researchers in 2015 [32]. These authors found that patients with leukemia were carriers of three inhibitory genes (2DL3, 2DL2 and $2 D L 4)$ significantly less often than healthy controls.

This study did not document a significant effect of KIR genotype on the risk of ALL. Slightly higher frequencies of some individual activating genes observed in patients with ALL do not necessarily mean that any of these genes are associated with increased risk of this malignancy. However, the risk of ALL may be higher in children who carry all 6 activating genes.

\section{The authors declare no conflict of interest.}

\section{References}

1. Kaatsch P (2010): Epidemiology of childhood cancer. Cancer Treat Rev 4: 277-285.

2. Kowalczyk JR, Dudkiewicz E, Balwierz W, et al. (2002): Incidence of childhood cancers in Poland in 1995-1999. Med Sci Monit 8: 587-590.

3. Hirsch B, Shimamura A, Moreau L et al. (2004): Association of biallelic BRCA2/FANCD1 mutations with spontaneous chromosomal instability and solid tumors of childhood. Blood 103: 2554-2559.

4. The International Nijmegen Breakage Syndrome Study Group (2000): Nijmegen breakage syndrome. Arch Dis Child 82: 400-406.

5. Derheimer FA Kastan MB (2010): Multiple roles of ATM in monitoring and maintaining DNA integrity. FEBS Lett 584: 3675-3681.

6. van der Burg, M Pac M, Berkowska MA, et al. (2010): Loss of juxtaposition of RAG-induced immunoglobulin DNA ends is implicated in the precursor B-cell differentiation defect in NBS patients. Blood 115: 4770-4777.

7. Hertzberg L, Vendramini E, Ganmore I, et al. (2010): Down syndrome acute lymphoblastic leukemia, a highly heterogeneous disease in which aberrant expression of CRLF2 is associated with mutated JAK2: a report from the International BFM Study Group. Blood 115: 1006-1017.

8. Chrzanowska KH, Piekutowska-Abramczuk D, Popowska E, et al. (2006): Carrier frequency of mutation 657del5 in the NBS1 gene in a population of Polish pediatric patients with sporadic lymphoid malignancies. Int J Cancer 118: 1269-1274.

9. Ljunggren HG, Kärre K (1990): In search of the 'missing self': MHC molecules and NK cell recognicion. Immunol Today 11: 237-244.

10. Kärre K (1992): An unexpected petition for pardon. Curr Biol 11: 613-615.

11. Lanier LL (2005): NK cell recognition. Annu Rev Immunol 23: $225-274$.

12. Parham P (2008): The genetic and evolutionary balances in human NK cell receptor diversity. Semin Immunol 20: 311-316.

13. Vilches C, Parham P (2002): KIR: diverse, rapidly evolving receptors of innate and adaptive immunity. Annu Rev Immunol 20: 17-51.

14. Traherne JA, Martin M, Ward R, et al. (2010): Mechanisms of copy number variation and hybrid gene formation in the KIR immune gene complex. Hum Mol Genet 19: 737-751.

15. Middleton D, Meenagh A, Gourraud PA (2007): KIR haplotype content at the allele level in 77 Northern Irish families. Immunogenetics 59: 145-158. 
16. Middleton D, Gonzelez F (2010): The extensive polymorphism of KIR genes. Immunology 129: 8-19.

17. Martin AM, Freitas EM, Witt CS, Christiansen FT (2000): The genomic organization and evolution of the natural killer immunoglobulin-like receptor (KIR) gene cluster. Immunogenetics 51: 268-280.

18. Khakoo SI, Rajalingam R, Shum BP, et al. (2000): Rapid evolution of NK cell receptor systems demonstrated by comparison of chimpanzees and humans. Immunity 12: 687-698.

19. Wilson MJ, Torkar M, Haude A, et al. (2000): Plasticity in the organization and sequences of human KIR/ILT gene families. Proc Natl Acad Sci U S A 97: 778-783.

20. Gourraud PA, Meenagh A, Cambon-Thomsen A, Middleton D (2010): Linkage disequili-brium organization of the human KIR superlocus: implications for KIR data analyses. Immunogenetics 62: 729-740.

21. QIAamp® DNA Mini and Blood Mini Handbook. Westburg BV, Leusden, The Netherlands 2007.

22. KIR Typing Kit. Miltanyi Biotec Inc. Auburn, USA.

23. Marsh SG, Parham P, Dupont B, et al. (2003): Killer-cell immunoglobulin-like receptor (KIR) nomenclature report, 2002. Immunogenetics 55: 220-226.

24. Schellekens J, Tilanus MG, Rozemuller EH (2008): The elucidation of KIR2DL4 gene polymorphism. Mol Immunol 45: 1900-1906.

25. Rajagopalan S, Fu J, Long EO (2001): Cutting edge: induction of IFN-gamma production but not cytotoxicity by the killer cell Ig-like receptor KIR2DL4 (CD158d) in resting NK cells. J Immunol 167: 1877-1881.

26. Gómez-Lozano N, Gardiner CM, Parham P, Vilches C (2002): Some human KIR haplotypes contain two KIR2DL5 genes: KIR2DL5A and KIR2DL5B. Immunogenetics 54: 314-319.

27. Graef T, Moesta AK, Norman PJ, et al. (2009): KIR2DS4 is a product of gene conversion with KIR3DL2 that introduced specificity for HLA-A*11 while diminishing avidity for HLA-C. J Exp Med 206: 557-572.

28. http://www.allelefrequencies.net (last update 30.11.2018)

29. Łuszczek W, Majorczyk E, Nowak I, et al. (2006): Inhibitory and activatory KIR gene frequencies in the Polish population. Int J Immunogenet 33: 167-170.

30. Almalte Z, Samarani S, Iannello A, et al. (2011): Novel association between activating killer-cell immunoglobulin-like receptor genes and childhood leukemia. Blood 118: 1323-1328.

31. Leung W (2011): Use of NK cell activity in cure by transplant. Br J Haematol 155: 14-29.

32. Misra MK, Prakash S, Moulik NR, et al. (2016): Genetic associations of killer immunoglobulin like receptors and class I human leukocyte antigens on childhood acute lymphoblastic leukemia among north Indians. Hum Immunol 77: 41-46.

33. Oevermann L, Firnkorn M, Michaelis S, et al. (2015): No association between the presence of killer-cell immunoglobulin-like receptor genes and susceptibility to childhood ALL. Blood 125: 3355-3357.

34. de Smith AJ, Walsh KM, Ladner MB, et al. (2014): The role of KIR genes and their cognate HLA class I ligands in childhood acute lymphoblastic leukemia. Blood 123: 2497-2503.

35. Nowak J, Kościńska K, Mika-Witkowska R, et al. (2015): Role of donor activating KIR-HLA ligand-mediated NK cell education status in control of malignancy in hematopoietic cell transplant recipients. Biol Blood Marrow Transplant 21: 829-839.

36. Babor F, Manser A, Schönberg K, et al. (2012): Lack of association between KIR genes and acute lymphoblastic leukemia in children. Blood 13: 2770-2772. 